ISSN 0001-6002/2002/44/4/160-162

A cta M édica Costarricense, $@ 2002$

Colegio de M édicos y Cirujanos

Caso Clínico

\title{
Edema pulmonar postobstructivo: reporte de 3 casos
}

\author{
Juan Ignacio-Padilla ${ }^{1}$, Donato Salas-Segura², Suen Kwoh-Sánchez ${ }^{3}$
}

\begin{abstract}
Resumen: El edema pulmonar postobstructivo (EPPO) es una complicación postoperatoria cuyo manejo adecuado depende de un diagnóstico etiológico correcto. EI EPPO se clasifica en dos tipos. El tipo I es secundario a la obstrucción aguda de la vía aérea superior. Por su parte, el tipo II ocurre luego de corregir quirúrgicamente una obstrucción crónica de la vía aérea. Fisiopatológicamente, el mecanismo que explica el cuadro es una disminución marcada de la presión intersticial en el nivel pericapilar pul monar resultante de la inspiración forzada. Lo anterior produce fuga capilar y el consecuente edema de pulmón. El manejo de este cuadro requiere de soporte respiratorio que incluye ventilación mecánica e incluso presión positiva al final de la espiración (PEEP). EI pronóstico es muy bueno si el diagnóstico es correcto y oportuno. Se reportan 3 casos que ilustran la entidad, su diagnóstico y manejo.
\end{abstract}

Descriptores: Edema pulmonar postobstructivo, complicación postoperatoria

Recibido:14 de mayo, 2002.

Aceptado: 13 de agosto, 2002.

Al enumerar las principales complicaciones pulmonares postoperatorias, se incluyen, entre otras, las atelectasias, la neumonía, la obstrucción de la vía aérea y el edema pulmonar. En lo que respecta a este último, su gravedad puede oscilar entre una condición de fácil manejo y una complicación letal. En la mayoría de los casos el edema pulmonar se relaciona con una administración excesiva de fluidos o retención de los mismos ${ }^{1}$.

Sin embargo, existe un tipo de edema pulmonar, principalmente de ocurrencia postoperatoria, donde que el mecanismo fisiopatológico es distinto, es conocido como edema pulmonar postobstructivo (EPPO) o edema pulmonar a presión negativa.

Se describen a continuación tres casos que ilustran esta enfermedad y se discuten aspectos relevantes sobre su etiología, fisiopatología y tratamiento.

A breviaturas: EPPO, edema pulmonar postobstructivo; PEEP, presión positiva al final de la espiración; UCI, U nidad de Cuidados Intensivos.

Unidad de Cuidados Intensivos. Hospital R. A . Calderón Guardia

Residente M edicina Interna . Hospital R. A . Calderón Guardia

Residente A nestesiología, Hospital R. A. Calderón Guardia

Correspondencia: Donato A. Salas Segura. Sección de M edicina, Hospital Calderón Guardia. Apdo. Postal 10706-1000 San José, Costa Rica. E-mail: dasscom@sol.racsa.co.cr

\section{Caso 1}

Paciente masculino de 16 años, sin antecedentes médicos 0 quirúrgicos de importancia, quien había sido traído al servicio de urgencias por un antecedente de trauma escrotal reciente y por el cual había estado hospitalizado días atrás. En esta ocasión reingresó por persistencia de sintomatología local y se decidió realizar una cirugía exploradora. Recibe anestesia general y como hallazgos operatorios, se anota estallamiento testicular izquierdo, que requirió de orquidectomía. Cursa con una evolución transoperatoria sin eventualidades. Dado su estado de conciencia y patrón respiratorio regular fue extubado en el posperatorio inmediato. Sin embargo, pocos minutos después inicia con disnea severa asociada a estridor. La saturación de oxígeno medida por oximetría de pulso desciende por debajo de $90 \%$. Se decide sedarlo y relajarlo de inmediato para proceder a la reintubación y ventilación, por medios mecánicos. Se encuentró salida de líquido espumoso abundante por el tubo endotraqueal. A pesar de recibir una $\mathrm{FIO} 2$ de $100 \%$, la saturación no es adecuada y se procede a agregar $10 \mathrm{~cm}$. PEEP a 10 $\mathrm{cm} . \mathrm{H} 2 \mathrm{O}$. Se obtiene una radiografía portátil de tórax que muestra infiltrados alveol ares bilaterales. Se decide trasladar a la unidad de cuidados intensivos ( $\mathrm{UCI}$ ), donde se continúa su manejo ventilatorio. Durante las siguientes 48 horas en esta Unidad, se manejado con sedación, diuréticos y ventilación mecánica. Los índices de intercambio gaseoso mejoran y se logra extubar sin complicaciones. Para ese momento, la radiografía de tórax no muestra infiltrados. Es egresado de terapia intensiva y dos días después abandona el hospital sin secuelas. 


\section{Caso 2}

Paciente masculino de 14 años, había sido sometido a laparotomía exploratoria por una apendicitis aguda grado 1, pero con ruptura apendicular transoperatoria y contaminación subsecuente. Durante el postoperatorio inmediato presentó estridor laríngeo severo y luego un cuadro clínico de edema agudo de pulmón. Se reintubó y se colocó en ventilación mecánica trasladándose a la U CI para su manejo ventilatorio. Su evolución fue muy buena, con bajos niveles de PEEP ( 5 $\mathrm{cm}$ de $\mathrm{H} 20$ como máximo) y FIO2 (0.35). No le fueron administrados diuréticos. Dos días después de su ingreso a la Unidad se extubó sin complicaciones. El paciente recibió antibióticos solo por su problema abdominal, del cual evolucionó satisfactoriamente.

\section{Caso 3}

Paciente masculino de 22 años, sin antecedentes médicos de importancia, quien fue sometido a cirugía reconstructiva electiva de ambos maxilares, con el fin de corregir un trastorno de oclusión y mejorar su vía aérea superior. No hubo complicaciones transoperatorias y la recuperación anestésica fue rápida y satisfactoria, por lo que se extuba en sala de recuperación. A los pocos minutos inicia con disnea y dificultad respiratoria, con aumento de secreciones traqueobronquiales y desaturación arterial, por lo que se intuba de nuevo.

La radiografía de tórax mostró infiltrados algodonosos difusos, característicos de edema pulmonar. El paciente es trasladado a la $\mathrm{UCl}$, para manejo ventilatorio de su edema pulmonar, y 48 horas después es extubado sin complicaciones, luego de que el edema resolviera completamente sin el uso de diuréticos. No hubo otras complicaciones y el resto de evolución del paciente fue satisfactorio.

\section{Discusión}

El edema pulmonar postobstructivo (EPPO) fue inicialmente descrito en perros, en $1927^{2}$. Fue hasta 1973 cuando Capitanio reporta, por primera vez, casos en humanos ${ }^{3}$. En 1977, Travis describe la entidad en pacientes pediátricos, con obstrucción de la vía aérea superior secundaria a epiglotitis ${ }^{4}$, y ese mismo año Oswalt et al., reportan los primeros tres casos en adultos 5 .

Se clasifica el EPPO en dos tipos: EPPO tipo I y EPPO tipo II ${ }^{6}$. En el primer caso, la causa de la obstrucción de la vía aérea superior es aguda e incluye como causas el laringospasmo postextubación, epiglotitis, cuerpos extraños, estrangulación, obstrucción de tubo endotraqueal, parálisis postoperatoria de cuerda vocal y síndrome de casi ahogado. En el tipo II, la causa de la obstrucción es crónica y se enumeran, entre otras condiciones asociadas, adenoidectomía, estenosis de coanas y postamigdalectomía.

El mecanismo fisiopatológico que explica la aparición de este tipo de edema pulmonar se basa en el siguiente fenómeno hemodinámico: al producirse una obstrucción de la vía aérea superior, la respuesta refleja es una esfuerzo inspiratorio forzado (maniobra de M ueller) ' 7 Stuth et al describen cómo, la aparición de hipo durante una extubación, puede producir un fenómeno similar a esta maniobra, desencadenando de igual forma el EPPO ${ }^{8}$. Como resultado de esto, se generan presiones negativas, particularmente bajas, en la caja torácica, que son transmitidas hasta el intersticio pericapilar pulmonar. Esto induce una fuga masiva de fluidos $y$, por ende, el edema pulmonar. Se han propuesto otros mecanismos asociados, como lo es el aumento marcado del retorno venoso; desplazamiento del septo interventricular hacia la izquierda, con el compromiso de la distensibilidad del ventrículo izquierdo ${ }^{9}$, y vasoconstricción pulmonar inducida por hipoxia.

A ún cuando estos mecanismos explican el tipo I, no son totalmente válidos para justificar el otro tipo. Se ha propuesto que el en tipo II, una obstrucción crónica de la vía aérea superior, como una hipertrofia adenoidea o una atresia de coanas, puede generar una presión positiva endógena al final de la espiración. Luego del procedimiento correctivo, se pierde este efecto, se desencadena la fuga capilar ${ }^{6}$. EI caso clínico 3 ilustra un ejemplo de EPPO tipo II. Independientemente del tipo, las manifestaciones del cuadro son súbitas y aparecen después de la resolución de la obstrucción, e incluyen los hallazgos propios de un edema pulmonar, como: disnea, cianosis, expulsión de material espumoso y rosado por la vía aérea, y deterioro del intercambio gaseoso, todos ellos descritos en los casos presentados. En algunos se ha descrito la aparición del edema, incluso antes de haber resuelto la causa de la obstrucción, haciendo pensar en un mecanismo de obstrucción variable ${ }^{9}$. El deterioro en la oxigenación suele ser realmente severo, y lleva a saturaciones de oxígeno, menores de $90 \%$ e índices de presión parcial de oxígeno arterial y fracción inspirada de oxígeno menores de 200. Desde el punto de vista radiológico, son característicos los infil trados al godonosos bilaterales. En una de las recopilaciones más amplias de casos de EPPO, Wilms y Shure reportan que 11 de 26 de los casos se debieron a laringospasmo ${ }^{10}$. El tiempo de instauración del cuadro de edema osciló desde un inicio inmediato hasta las 6 horas posteriores. En todos aquellos casos en los que se realizaron mediciones hemodinámicas invasivas, la presión de oclusión de arteria pulmonar fue normal ${ }^{10}$.

La forma más severa del cuadro es la hemorragia alveolar difusa ${ }^{7,11}$. Si bien se describe clásicamente esta forma como de predominio parenquimatoso, se ha relacionado la entidad con hemorragia originada en la vía aérea ${ }^{12}$. Uno de los riesgos más importante es confundir esta entidad con otras causas de hemorragia pulmonar, como las de origen autoinmune, sometiendo al paciente a terapias inmunosupresoras riesgosas. Cabe mencionar que en el tercer caso que aquí reportamos, el paciente presentó sangrado profuso a través del tubo endotraqueal. Esta hemorragia cedió espontáneamente al mejorar su condición pulmonar. 
No se ha establecido alguna relación directa con un esquema anestésico dado. No obstante, algunos reportes pretenden asociar el uso de opiáceos con la entidad, a raíz del fenómeno conocido, de que el estímulo de receptores mu de opioides produce rigidez torácica, glótica 0 ambas ${ }^{13,14}$. Es de interés mencionar que uno de los primeros reportes de edema pulmonar asociado a opiáceos (buprenorfina) se interpretó, probablemente, de manera errónea, como una reacción alérgica ${ }^{14}$.

El abordaje de este tipo de edema pulmonar depende de sospechar su etiología y hacer un adecuado diagnóstico diferencial. La presencia de factores predisponentes (trauma, sepsis o transfusiones masivas), infiltrados pulmonares bilate-rales y deterioro del intercambio gaseoso previo a la obstrucción, deben hacer pensar más bien en edema pulmonar lesional, cuyo manejo y pronóstico es diferente. Por otra parte, en presencia de sobrecarga de fluidos, en especial en pacientes con reserva ventricular limitada, debe sospecharse un edema de origen hidrostático.

El soporte respiratorio debe buscar una oxigenación óptima, recurriendo en la mayoría de los casos al uso de ventilación mecánica asistida. A unque no existe un protocolo definido para el manejo de estos casos, es frecuente la necesidad de usar PEE P. En aquellos casos en los que el deterioro del intercambio gaseoso es muy severo, se debe recurrir a sedación y hasta relajación con bloqueadores de tipo neuromuscular. EI uso de diuréticos es frecuente, pero no hay evidencia suficiente para considerar su empleo rutinario. El pronóstico de EPPO es muy bueno, y en la mayoría de los casos se resuelve en un plazo no mayor a 48 horas ${ }^{15}$.

En conclusión, el edema pulmonar postobstructivo es una complicación, especialmente postoperatoria, que aunque no es muy frecuente, debe ser sospechada en aquellos pacientes con al gunos factores de riesgo. A unque su pronóstico es muy bueno, un abordaje equivocado puede ser letal.

\section{Abstract}

Postobstructive pulmonary edema (POPPE) is a postoperative complication and its approach depends on an appropriate diagnosis. POPPE can be classified in two types. POPPE type I is caused by acute upper airway obstruction, and type II is due to chronic obstruction. The mechanism of this entity is a marked decrease in pericapilar interstitical pressure in the lung that results in capillary leakage and edema. Its treatment requires mechanical ventilatory support including positive end expiratory pressure (PEEP). Its prognosis is very good if the diagnosis is done appropriately. We report three cases that illustrate its diagnosis and treatment.

\section{Referencias}

1 A rieff, A .I. Fatal Postoperative Pulmonary Edema. Pathogenesis and Literature Review. Chest 1999; 115: 1371-1377

2 M oore RL. The response to respiratory resistance: a comparison of the effects produced by partial obstruction in the inspiratory and expiratory phases of respiration. J Exp M ed 1927;45:1065-80.

3. Capitanio MA, Kirkpatrick JA. Obstructions of the upper airway in children as reflected on the chest radiograph. Radiology 1973;107:15961. Citation

4. Travis KW, Todres ID, Shannon DC. Pulmonary edema associated with croup and epiglottitis. Pediatrics 1977;59:695-698

5 Oswalt C, Gates GA, Holmstrom M G. Pulmonary edema as a complication of acute airway obstruction. J A M A 1977; 34: 1833-1835

6 Van Kooy M A, Gargiulo RF. Postobstructive pulmonary edema. Am Fam Physician 2000; 62 : 401-4.

7 Schwartz D. Maroo A. M alhorta A. K esselman H. Negative Pressure Pulmonary Hemorrhage. Chest 1999; 115: 1194-1197

8 Stuth E. Strucke A. B erens R. N egative-pressure Pulmonay Edema in a Child with Hiccups during Induction. A nesthesiology 2000; 93: 282284

9 Masa Jiménez J. F. Verea Hernando H. De la Cruz Ríos J. Sánchez J. Pulmonary Edema Due to U pper A irway Obstruction. Chest 1990; 97 : 255

10 Willms, D. Shure, D. Pulmonary Edema due to Upper Airway Obstruction in A dults. Chest 1988; 94: 1090-1092

11 Dolinski S., MacGregor D. Scuderi P. Pulmonary Hemorrhage A ssociated with Negative Pressure Pulmonary Edema. A nesthesiology 2000, 93; 888-890

12 M cConkey P. A irway bleeding in negative-pressure pulmonary edema. A nesthesiology 2001;95:272

13 A randia. H, Patil V. U. Glottic Closure Following Large Doses of Fentanyl. A nesthesiology 1987, 66: 575-576

14 Gould, D. Buprenorphine Causes Pulmonary Edema Just Like Other M u-opioid Narcotics. Chest 1995; 107: 1478-1479

15 Gómez R, Domínguez A, Herranz-A ndrés, et al. Dos casos de edema agudo de pulmón secundario a laringospasmo. Rev. Esp. A nestesiol. Reanim 1999; 46: 317-319 